\title{
Silicon-based organic-inorganic microcavity and its dispersion curve from angle-resolved photoluminescence
}

\author{
A. Arena, S. Patanè, G. Saitta, S. Savasta, and R. Girlanda ${ }^{\text {a) }}$ \\ INFM and Dipartimento di Fisica della Materia e Tecnologie Fisiche Avanzate, Università di Messina, \\ Salita Sperone, 31, I-98166 Sant'Agata-Messina, Italy \\ R. Rinaldi \\ INFM and Dipartimento di Scienza dei Materiali, Università di Lecce, via Arnesano, I-73100 Lecce, Italy
}

\begin{abstract}
We report an experimental study of a planar organic-inorganic microcavity consisting of a porous silicon distributed Bragg reflector, a single layer of acridina orange as active material and a top aluminum reflector. By tuning the cavity resonance energy around the maximum of the organic material photoluminescence, we found an intense emission and a spectral narrowing of the emission band to about $45 \mathrm{meV}$. The angle-resolved photoluminescence spectra enable us to determine the microcavity dispersion curve. The very good agreement with the theoretical dispersion provides a precise determination of the refractive index of the organic material.
\end{abstract}

[S0003-6951(98)01120-6]

In the last few years the study of light confinement in solid state microcavities has attracted many researchers due to the possibility of theoretical and experimental investigations in the aim of realizing novel optical and electro-optical devices. $^{1-3}$ Electromagnetic field confining devices can strongly modify the optical properties of the emitting material thus determining new radiative phenomena and permitting the control of the spectral properties of emission. Microcavities are now fabricated in a wide variety of solid state systems, including semiconductors and organic materials; the simple planar geometry of the Fabry-Pérot is the most widely used for realizing solid state microcavities. Quantum wells embedded in high $\mathrm{Q}$ planar microcavities with mirrors constituted by distributed Bragg reflectors (DBRs) showed noticeable modifications of spontaneous emission and cavity quantum electrodynamic effects. ${ }^{4,5}$ Cavity-embedded quantum wells are usually produced by expansive and time consuming methods as molecular beam epitaxy. Alternative methods have been proposed to realize light-emitting microcavities, for instance, using alternative materials such as porous silicon ${ }^{6-9}$ and/or organic materials. ${ }^{10-12}$ The main attractive features of these devices are the low cost, the relatively simple methods of preparation, the wide range of tunability and the innovative working principles; nevertheless, prototypes suffer low reproducibility, low efficiency, and short life. Intensive studies on the optical properties of porous silicon (PS) have been carried out with the aim of obtaining Si-based integrated microphotonic devices. ${ }^{13}$ Also there have been intense research activities in the field of organic light-emitting devices. ${ }^{14,15}$ The use of polymeric and organic materials as active media is of course a fascinating target in designing novel optoelectronic devices, due to high quantum efficiency, tunability, and to the possibility of engineering their optical and electronic features by changing or adding a host specie in the main organic matrix.

${ }^{a)}$ Electronic mail: girlanda@imeuniv.unime.it
In the present work we show a simple, inexpensive and reproducible method to realize a hybrid organic-inorganic optical microcavity grown on a Si wafer, where the organic layer is the active one, while the mirrors are realized by using a bottom porous silicon DBR and a top metal thin film. The porous silicon-organic-metal microcavity, because of low cost and ease of integration with existing semiconductor technology, is expected to allow in future the realization of electro-optical devices such as modulators and highefficiency light-emitting diodes in a wide energy range simply changing the cavity parameters and/or the organic material.

In Fig. 1 the structure of the planar organic-inorganic microcavity and the experimental geometry are shown. The first step to fabricate the planar microcavity consists in realizing the bottom PS DBR. In the last few years a considerable amount of papers have reported the realization of DBRs from a silicon surface. ${ }^{6-9}$ The layers of different refractive index composing DBRs can be obtained periodically changing the etching parameters, usually the current or the illumination of the sample. ${ }^{16}$ Here we started from a single-crystal, 111-oriented of B-doped Si wafer. Anodization was carried out in an electrochemical cell filled with a solution of $48 \%$ HF: ethanol $=1: 1$ and the current was periodically tuned by means of a computer-controlled current generator. Our PS

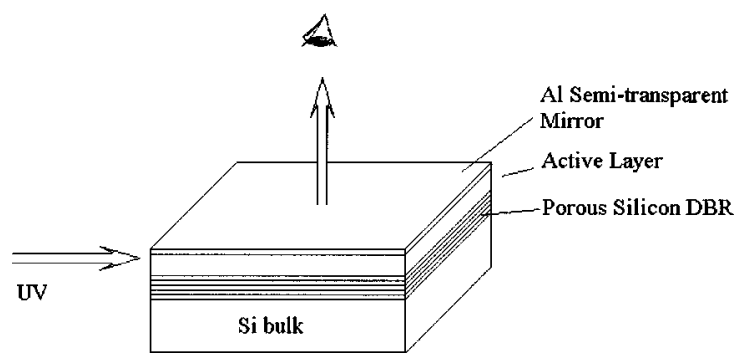

FIG. 1. Schematic structure of the planar organic-inorganic microcavity and geometry of the experiment. 

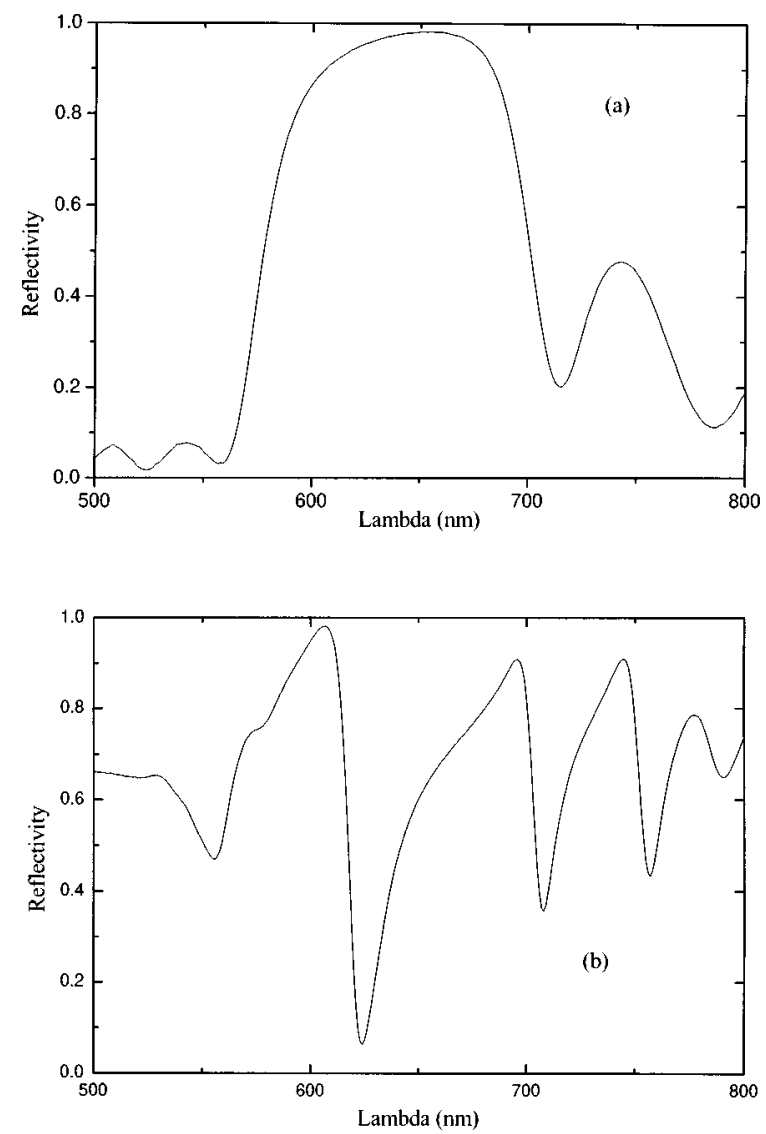

FIG. 2. (a) Room temperature reflectivity spectrum of the porous silicon DBR. (b) Room temperature reflectivity spectrum of the porous siliconorganic-metal microcavity.

mirror is composed by ten pairs of alternating PS $\lambda / 4$ layers with refractive index of about 1.5 and 2.5, thickness of about $110 \mathrm{~nm}$ and $53 \mathrm{~nm}$ obtained, respectively, with a density current-time ratio of $3 \mathrm{~mA} / \mathrm{cm}^{2}-8.0 \mathrm{~s}$. and $70 \mathrm{~mA} / \mathrm{cm}^{2}-0.5 \mathrm{~s}$. The DBR room temperature (RT) reflectivity spectrum is shown in Fig. 2(a). The stop band is about $100 \mathrm{~nm}$ wide and the maximum reflectivity is about $98 \%$ at $650 \mathrm{~nm}$.

The organic layer was obtained evaporating the material in high vacuum by using a homemade stainless steel crucible whose temperature was monitored by a thermocouple and manually controlled, changing the current flow in a tungsten filament. The choice of the organic material is driven by features as the emission energy, the evaporation or sublimation temperature, the capacity to evaporate without decomposing, and the photoluminescence (PL) yield. A wide spectra of possibilities are available from commercial resources: acridine orange is a low-cost dye-laser material, ${ }^{17}$ whose melting point is sufficiently low and which permits the realization of thin films with good optical surfaces. The high vacuum chamber was equipped with an INFICON Leybold thin-film deposition controller to monitor the thickness of the organic and metallic layers onto the PS mirror. The control of the evaporation rate is one of the most important steps of the growing process, in particular a low and uniform deposition rate is important to avoid molecular decomposition and to ensure the goodness of surfaces. Our organic layer was grown in $1 \mathrm{~h}$ with a thickness of about $400 \mathrm{~nm}$, while the pressure of the chamber during the evaporation was lower than $2 \times 10^{-6}$ Torr. Finally an Al layer $12 \mathrm{~nm}$ thick
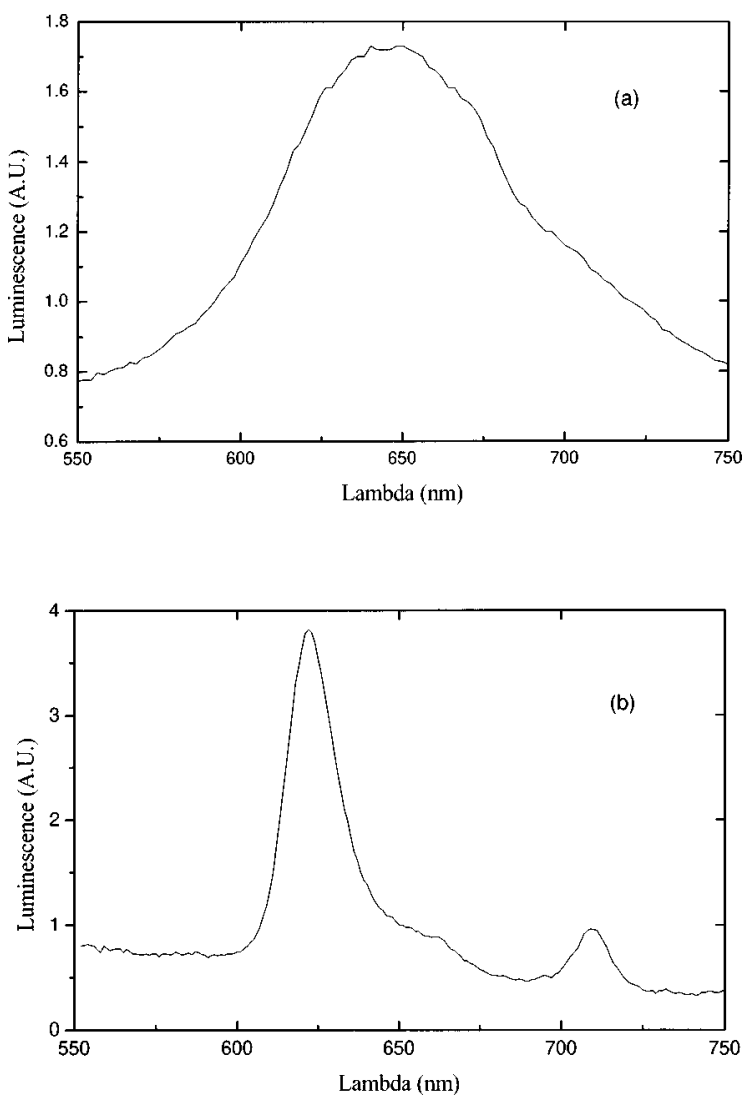

FIG. 3. (a) Room temperature photoluminescence spectrum of a thick layer of acridina orange deposited onto a silicon substrate. (b) Room temperature photoluminescence spectrum of the porous silicon-organic-metal microcavity whose reflectivity is shown in Fig. 2(b).

was deposited to realize the top mirror of the cavity.

The optical reflectivity of the sample was investigated by means of a Perkin-Elmer Lambda 2 UV/VIS spectrophotometer equipped with two specular reflectance accessories. Room temperature luminescence was recorded by a computer-controlled Perkin-Elmer 650-40 fluorescence spectrophotometer. The sample was stored for one month in air at RT showing no modification of its features.

The reflectivity at normal incidence of the PS DBR and of the PS-organic-metal microcavity are shown in Fig. 2. The cavity effect can be directly observed by comparing these two spectra: the first one shows the classical optical response of a DBR with a very high flat reflectivity; the last one is clearly characterized by a deep peak centered at about 620 and $20 \mathrm{~nm}$ wide. By using the standard transfer matrix approach, ${ }^{18}$ we find a theoretical value $\Delta \lambda_{r}$ of about $22 \mathrm{~nm}$ in good agreement with the experimental value. Fig. 3(a) shows the room-temperature PL spectrum obtained by exciting with a $400 \mathrm{~nm}$ beam a thick film of the organic material deposited onto a silicon substrate. We find a PL band centered at about $650 \mathrm{~nm}$ and about $150 \mathrm{~nm}$ wide. Figure 3(b) shows the cavity emission in the forward direction. We observe dramatic changes in the spectral response showing a strong narrowing of the PL emission. We also observe an increase of a factor of about 3 of the PL peak; unfortunately the experimental setup does not permit a precise evaluation of the enhancement. The spectral width of the emission results further narrowed to $\Delta \lambda_{e}=18 \mathrm{~nm}$ with respect to the reflectivity dip; the quality factor $Q=\lambda / \Delta \lambda$, is about 34 . 
The main reason the quality factor is limited is because of the reflectivity of the thin $\mathrm{Al}$ mirror more than the reflectivity of the porous silicon mirror or the roughness of the organic layer. This can be shown by inserting the reflectivity $R_{1}=0.98$ and $R_{2} \approx 0.55$ (for a wave escaping from the cavity) of the PS DBR and of the Al mirror in the theoretical approximated full width at half maximum $(\mathrm{FWHM})^{18}$

$$
\Delta \lambda=\frac{\lambda^{2}}{2 n d} \frac{1-\sqrt{R_{1} R_{2}}}{\pi\left(R_{1} R_{2}\right)^{1 / 4}},
$$

where $d$ and $n$ are the thickness and the refractive index of the organic layer, respectively. Moreover, our preliminary studies on these devices show that lowering the pressure and the deposition rate of the organic thin film increases the efficiency of emission and permits a better control of the thickness of the active layer.

We have also performed angle-resolved PL spectra. In the weak-coupling regime the PL resonance condition is determined by the dispersion of the cavity mode $E\left(\mathbf{k}_{\|}\right)$which has the usual dependence

$$
E\left(\mathbf{k}_{\|}\right)=\sqrt{E_{0}^{2}+c^{2} \hbar^{2} k_{\|}^{2} / n^{2}},
$$

where $n$ is the refractive index of the cavity and $k_{\|}$ $=\omega \sin \left(\theta_{\text {out }}\right) / c$ is the modulus of the in-plane wave vector, being $\theta_{\text {out }}$ the external observation angle, and $E_{0}$ is the resonance energy of the cavity at normal incidence. The measurement of angle-resolved photoluminescence spectra is thus an efficient and simple tool to measure the microcavity dispersion in a wide angular range. Fitting the experimental data by using Eq. (2), it is possible to make a precise determination of the refractive index of the organic material. In Fig. 4 we report the PL angle-resolved resonance energies of the microcavity and the best fit of experimental data (continuous line); the obtained value for the organic cavity refractive index is $n=2.35$. We observe that, due to the functional dependence of $\lambda$ on $n$ and $\theta$, the fitting procedure results in a quite precise determination of the refractive index. This statement is clearly justified observing the dotted curve in Fig. 4 representing the dispersion of the microcavity calculated by Eq. (2) with a refractive index of 2.0.

We have realized an organic-inorganic planar microcavity grown on a $\mathrm{Si}$ wafer, thus exploring the possibility of realizing versatile Si-based light-emitting devices. The microcavity consists of a PS DBR as bottom mirror, a thin film of acridine orange as active layer and a top aluminum mirror. Reflectivity measurements show cavity effects. A noticeable narrowing of the active layer emission band is observed in PL spectra. We have determined the microcavity dispersion curve by angle-resolved PL spectra and the very good agreement with the theoretical Fabry-Pérot dispersion provides a precise determination of the refractive index of the organic

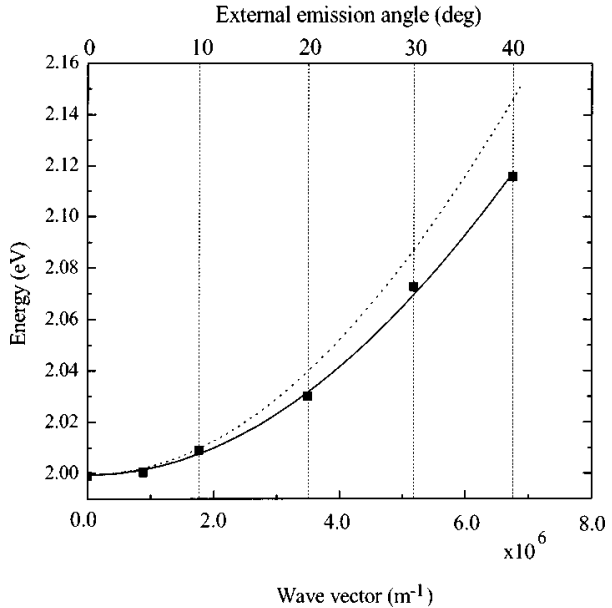

FIG. 4. Microcavity dispersion curve, deduced from angle-resolved photoluminescence measurements. The continuous line is the best fit of the experimental data evaluated using Eq. (2). The dotted line is the theoretical dispersion curve evaluated with $n=2.0$.

material. High quantum efficiency, a high degree of tunability and strong nonlinearities of polymeric and molecular materials, together with microcavity effects and ease of integration of Si and PS with the existing electronic technology, are the ingredients which make these hybrid microcavities promising for the future realization of electro-optical devices such as modulators and high-efficiency light-emitting diodes in a wide energy range.

${ }^{1}$ Y. Yamamoto, S. Machida, and G. Bjork, Phys. Rev. A 44, 657 (1991).

${ }^{2}$ Y. Yamamoto and R. E. Slusher, Phys. Today 46, 66 (1993).

${ }^{3}$ R. E. Slusher and C. Weisbuch, Solid State Commun. 92, 149 (1994).

${ }^{4}$ C. Weisbuch, M. Nishioka, A. Ishikawa, and Y. Arakawa, Phys. Rev. Lett. 69, 3314 (1992).

${ }^{5}$ T. B. Norris, J. K. Rhee, C. Y. Sung, Y. Arakawa, M. Nishioka, and C. Weisbuch, Phys. Rev. B 50, 14663 (1994).

${ }^{6}$ V. Pellegrini, A. Tredicucci, C. Mazzoleni, and L. Pavesi, Phys. Rev. B 52, 14328 (1995).

${ }^{7}$ L. Pavesi, R. Guardini, and C. Mazzoleni, Solid State Commun. 97, 1051 (1996).

${ }^{8}$ M. Araki, H. Koyama, and N. Koshida, J. Appl. Phys. 80, 4841 (1996).

${ }^{9}$ M. Araki, H. Koyama, and N. Koshida, Appl. Phys. Lett. 69, 2956 (1996).

${ }^{10}$ B. Zhang, Y. Ling, Z. Xia, X. Ding, S. Wang, D. Zhou, and C. Huang, Solid State Commun. 97, 445 (1996).

${ }^{11}$ V. Cimrovà, and D. Neher, J. Appl. Phys. 79, 3299 (1996).

${ }^{12}$ J. Gruner, F. Cacialli, I. D. W. Samuel, and R. H. Friend, Synth. Met. 76, 137 (1996).

${ }^{13}$ E. F. Schubert, N. E. J. Hunt, M. Micovic, R. J. Malik, D. L. Sivco, A. Y. Cho, and G. J. Zhszik, Science 256, 943 (1994).

${ }^{14}$ V. Bulovic, G. Gu, P. E. Burrows, S. R. Forrest, and M. E. Thompson, Nature (London) 29, 380 (1996).

${ }^{15}$ Q. Pei, G. Yu, C. Zhang, Y. Yang, and A. J. Keeger, Science 269, 1086 (1995).

${ }^{16}$ L. Pavesi and P. Dubos, Semicond. Sci. Technol. 12, 570 (1997).

${ }^{17}$ Dye Lasers, edited by F. P. Schäfer (Springer, Berlin, 1973), p. 180.

${ }^{18}$ H. A. McLeod, Thin-Film Optical Filters (Hilger, Bristol, 1986), pp. 238 240. 